\title{
EVALUACIÓN DE LA PRODUCCIÓN CIENTÍFICA DE POSTGRADO EN ENFERMERÍA SEGÚN LOS INDICADORES DE SALUD ${ }^{1}$
}

\section{EVALUATION OF SCIENTIFIC PRODUCTION IN NURSING GRADUATE PROGRAM ACCORDING TO HEALTH INDICATORS}

\author{
Natália Gonçalves* \\ Rejane Kiyomi Furuya ${ }^{* *}$ \\ LIDIA APARECIDA Rossi ${ }^{* *}$ \\ Rosalina Aparecida Partezani Rodrigues ${ }^{* * * *}$ \\ Maria Lúcia do Carmo Cruz RobazzI ${ }^{* * * * *}$
}

\begin{abstract}
RESUMEN
Este estudio comparó la producción de disertaciones y tesis de postgrado de la Escuela de Enfermería de Ribeirão Preto de la Universidad de São Paulo (EERP-USP) con los indicadores nacionales de salud: mortalidad general y morbilidad hospitalaria, por grupos de causas. Fueron recolectadas informaciones acerca de las tesis y disertaciones de 2007-2009 y de los indicadores de 2007. Las producciones se clasificaron de acuerdo a los capítulos de la Clasificación Estadística Internacional de Enfermedades y Problemas Relacionados con la Salud (CIE 10). Se encontraron 334 publicaciones y tesis. Cinco capítulos de la CIE 10 fueron similares cuando se compararon los principales temas de las disertaciones y tesis con las causas de mortalidad; en relación a la morbilidad, ocho capítulos coincidieron. Los temas de las producciones de la EERP y los datos de mortalidad general y morbilidad hospitalaria se relacionaron. De este modo, las producciones están alineadas a las necesidades de la población, la práctica profesional y las políticas de salud.
\end{abstract}

Palabras clave: Indicadores de salud, investigación en enfermería, educación de postgrado en enfermería.

\begin{abstract}
This study compared the production of theses and dissertations from Graduate Programs at the University of São Paulo at Ribeirão Preto College of Nursing (EERP-USP) with national health indicators: general mortality and hospital morbidity, according to the groups of causes. Information about theses and dissertations between

\footnotetext{
${ }^{1}$ Artigo produzido na disciplina ERG 5894 - Educação da Pós-Graduação e Pesquisa em Saúde e Enfermagem do Programa Interunidades de Doutoramento em Enfermagem da Escola de Enfermagem (EE) e Escola de Enfermagem de Ribeirão Preto (EERP) da Universidade de São Paulo (USP).

* Enfermeira. Aluna do Programa Interunidades de Doutoramento em Enfermagem da EE e EERP da USP. EERP-USP, Centro Colaborador da Organização Mundial da Saúde para o desenvolvimento da pesquisa em enfermagem. Ribeirão Preto, São Paulo, Brasil. Email: nataliasjbv@hotmail.com

${ }^{* *}$ Enfermeira. Aluna do Programa Interunidades de Doutoramento em Enfermagem da EE e EERP da USP. EERP-USP, Centro Colaborador da Organização Mundial da Saúde para o desenvolvimento da pesquisa em enfermagem, Ribeirão Preto, São Paulo, Brasil. Email: re.furuya@gmail.com

${ }^{* * *}$ Enfermeira. Professor Departamento de Enfermagem Geral e Especializada (EGE) da EERP-USP, Centro Colaborador da OMS para o desenvolvimento da pesquisa em enfermagem, Ribeirão Preto, São Paulo, Brasil. Email: rizzardo@eerp.usp.br ${ }^{* * * *}$ Enfermeira. Professor do Departamento de EGE da EERP-USP, Centro Colaborador da OMS para o desenvolvimento da pesquisa em enfermagem, Ribeirão Preto, São Paulo, Brasil. Email: rosalina@eerp.usp.br

${ }_{* * * * *}$ Enfermeira. Professor do Departamento de EGE da EERP-USP, Centro Colaborador da OMS para o desenvolvimento da pesquisa em enfermagem, Ribeirão Preto, São Paulo, Brasil. Email: avrmlccr@eerp.usp.br
} 
2007 and 2009 and health indicators from 2007 were collected. Theses and dissertations were classified according to chapters of the International Statistical Classification of Diseases and Related Health Problems (ICD 10). In total, 334 dissertations and theses were found. Five chapters of the ICD 10 were similar when compared to the main themes of these productions and the causes of mortality. Regarding morbidity, eight chapters coincided. There was a relationship between the subjects of dissertations and theses produced by EERP and the data of general mortality and hospital morbidity. Thus, the production are related to the needs of the population, the professional practice and to the health policies.

Key words: Health status indicators, nursing research, education, nursing, graduate.

Fecha recepción: 29/06/12 Fecha aceptación:12/07/13

\section{INTRODUCCIÓN}

La enfermería es un área de la salud que viene creciendo respecto a la cualificación del proceso investigativo y al fortalecimiento de la profesión en Brasil. Se observa que la investigación acompaña esta evolución, con ampliación y fortalecimiento de los cursos de postgrado, grupos de investigación y recursos financieros destinados a la formación y producción de conocimiento (1).

La educación de postgrado en enfermería contribuye a la formación de recursos humanos calificados y al desarrollo científico del área en Brasil. Muestra posición de destaque en el contexto latinoamericano debido a la consolidación alcanzada en el país (2).

En Brasil, los programas de postgrado en enfermería empezaron en la década de los 70, con la creación del primer curso de maestría en 1972, por la Escuela de Enfermería Ana Neri. En la Escuela de Enfermería de Ribeirão Preto de la Universidad de São Paulo (EERP-USP), esa década fue marcada por la preparación y cualificación de los docentes para la educación de postgrado (3). Fueron creados los cursos de maestría para los dos departamentos existentes: Enfermería Psiquiátrica y Ciencias Humanas (1975) y Enfermería General y Especializada (1979). En los años de 1980, fue creado el primer programa de doctorado en enfermería, el Interunidades de Doctoramiento en Enfermería, fruto de un esfuerzo colectivo de la
Escuela de Enfermería de la Universidad de São Paulo (EE-USP) y de la Escuela de Enfermería de Ribeirão Preto de la Universidad de São Paulo (EERP-USP). A partir de 1988, la EERP-USP fue designada como Centro Colaborador de la Organización Mundial de la Salud para el Desarrollo de la Investigación en Enfermería, lo que apoyó el desarrollo de nuevos cursos de maestría y doctorado y en la formación de mayor cantidad de profesionales calificados (3).

Hoy día, en la EERP-USP existen cuatro programas de postgrado: Enfermería Fundamental, Enfermería en Salud Pública, Enfermería Psiquiátrica e Interunidades de Doctoramiento en Enfermería, siendo el último desarrollado en colaboración con la EE-USP.

En la reciente evaluación llevada a cabo por la Coordinación de Perfeccionamiento de Personal de Nivel Superior (CAPES), la nota de los Programas Enfermería Fundamental y Enfermería en Salud Pública fue seis y la de los Programas Enfermería Psiquiátrica e Interunidades de Doctoramiento en Enfermería continuó en cinco (4). Esos datos evidencian que los programas vinculados a la EERP-USP son de excelente calidad y pueden ser considerados como referencia para la educación de postgrado en enfermería en Brasil.

Se sabe que los programas de postgrado en enfermería permiten la formación de recursos humanos en investigación avanzada, indicando las necesidades de la práctica y ampliando los conocimientos en favor de la 
mejora de la salud y calidad de vida de la población $(1,5)$. Sin embargo, no hay estudios que analicen la producción de postgrado respecto a los indicadores de salud.

Las informaciones y los indicadores de salud han sido descritos como los "ojos" de los responsables por la formulación de las políticas de salud (6). Los indicadores de salud, como la mortalidad general y la morbilidad hospitalaria fueron desarrollados para facilitar la cuantificación y evaluación de las informaciones producidas. Contiene informaciones relevantes sobre determinados atributos, dimensiones del estado de salud y desempeño del sistema de salud. El análisis de esos indicadores puede ser por local de ocurrencia, sexo, rango de edad, grupos de causas y específica para determinada enfermedad (7). Así, los indicadores pueden ayudar en el direccionamiento de las investigaciones en el área de salud pública, incluso la enfermería. El conocimiento de las informaciones generales de salud de una nación contribuye al desarrollo de investigaciones en áreas con peores indicadores. Las informaciones generales sobre salud también orientan la atención en salud y el cuidado de enfermería con enfoque del cuidado en áreas con mayor morbilidad y mortalidad.

El conocimiento de la producción científica en el nivel de postgrado en enfermería es importante para evaluar la contribución de esa formación en la práctica asistencial y en la educación en salud y enfermería. En un estudio sobre la producción de conocimiento en un curso de maestría en Enfermería en Brasil, los resultados evidenciaron la consolidación de conocimiento con enfoque en el cuidado a la salud y en la educación (8).

Considerando que la producción del conocimiento debe ejercer impacto positivo en las necesidades sociales (regionales, nacionales e internacionales) y que la EERP-USP se destaca en Brasil como institución de enseñanza e investigación, la finalidad del presente estudio fue comparar la producción de las disertaciones y tesis de los programas de postgrado de la EERP-USP con los indicadores de salud nacional: mortalidad general y morbilidad hospitalaria, de acuerdo con los grupos de causas. La realización de este estudio se justifica por la inexistencia de investigación que tratan de esa temática, y también por la importancia de desarrollar investigaciones de acuerdo con los datos epidemiológicos del país.

\section{MATERIAL Y MÉTODO}

Investigación de diseño cuantitativo no experimental, del tipo descriptivo exploratorio (9).

Los datos de las disertaciones y tesis de los programas de postgrado de la EERP-USP en el período 2007 - 2009 fueron encontrados en los Cuadernos de Indicadores de la CAPES (10), disponibles en www.capes.gov.br/ avaliação. Tales documentos contienen informaciones completadas anualmente por los programas, enviadas a la CAPES y utilizadas en el proceso de evaluación de los programas de postgrado. Hoy día, los Cuadernos abarcan a 11 documentos. Para este estudio fue utilizado el documento "Tesis y Disertaciones", que comprende las siguientes informaciones: autor, título, volúmenes, número de páginas, idioma, orientador, área de concentración, línea de investigación, proyecto de investigación, mesa examinadora y financiador (10).

A partir de la lectura del título y resumen, las disertaciones y tesis fueron agrupadas de acuerdo con los capítulos de la Clasificación Estadística Internacional de Enfermedades y Problemas Relacionados con la Salud (CIE 10) (11). Se extrajeron los siguientes datos de las tesis y disertaciones: número de identificación, autor, título, clasificación según capítulo de CIE 10, área de concentración, línea de investigación, proyecto de investigación, maestría, doctorado, año, financiamiento, programa al cual pertenecía el trabajo.

Para el análisis de las distribuciones de 
mortalidad general y morbilidad hospitalaria en Brasil ocurridas en el 2007, según los grupos de causas, fue utilizada la base de datos del Departamento de Informática del Sistema Único de Salud (DATASUS) (12).

Los datos de mortalidad general son registrados en el Sistema de Informaciones sobre Mortalidad (SIM). Ese sistema está implantado en todo el territorio nacional y registra los fallecimientos ocurridos en el país, utilizando como fuente la declaración de fallecimiento (12). Los datos de mortalidad permiten inferencias sobre las condiciones de salud de la población (13).

La mortalidad proporcional por grupos de causas es la distribución porcentual de fallecimientos por grupos de causas, en una población, según el local y año. Este indicador mide la participación relativa de los principales grupos de causas de muerte (de acuerdo con el capítulo de la CIE 10) en el total de muertes con causa definida (7).

La morbilidad proporcional por grupos de causas es el porcentaje de las internaciones hospitalarias por el Sistema Único de Salud (SUS), por grupos de causas, en una población, según local y año (7).

Las informaciones sobre mortalidad general y morbilidad hospitalaria son importantes para identificar situaciones de desigualdad y tendencias que demandan acciones y estudios específicos; desarrollar análisis comparativos de la concentración de recursos médico-hospitalarios; apoyar procesos de planificación, gestión y evaluación de políticas públicas dirigidas a la atención hospitalaria y medidas preventivas; evaluar los niveles de salud y desarrollo socioeconómico de la población para comparaciones nacionales e internacionales (7).

Las informaciones recolectadas fueron organizadas en plantillas del software Microsoft Excel 2007 y fueron desarrollados análisis de estadística descriptiva (frecuencia simple y relativa) para las variables de interés: cantidad de disertaciones y tesis desarrolladas entre 2007 y 2009, y los datos de mortali- dad general y morbilidad hospitalaria según las causas de la CIE 10.

Como el estudio utilizó exclusivamente datos de dominio público que no identifican los participantes de la investigación, sin involucrar a seres humanos, no fue necesaria la aprobación por el Comité de Ética en Investigación, de acuerdo con las orientaciones de la Comisión Nacional de Ética en Investigación (14).

\section{RESULTADOS}

En el período 2007 - 2009 fueron defendidas 334 disertaciones y tesis en los programas de postgrado de la EERP-USP. En ese grupo, $193(57,8 \%)$ eran disertaciones y 141 $(42,2 \%)$ tesis.

De las 334 disertaciones y tesis, 99 (29,6\%) no estaban relacionadas con cualquier capítulo de la CIE 10. Los temas de esas producciones fueron: gestión 13, enseñanza 13, ancianos 11, actuación del equipo de enfermería 7, administración de medicamentos 6 , urgencia y emergencia 6 , políticas de salud 5 , humanización 5, estrategia salud de la familia 5, desarrollo y validación de cuestionarios 5 , sistematización de la atención de enfermería 3 , atención primaria 3 , medio ambiente 3 , centro quirúrgico 2 , control de infección 2 , ética y bioética 2 , arteterapia 2 , aceptabilidad y satisfacción del usuario 2, estudio de laboratorio 1, desarrollo de software 1, accesibilidad del servicio 1, actuación interprofesional 1.

Los principales temas de las disertaciones y tesis, según la CIE 10, fueron: factores que afectan el estado sanitario y el contacto con los servicios de salud (capítulo XXI), trastornos mentales y de comportamiento (capítulo V) y enfermedades endocrinas, nutricionales y metabólicas (capítulo IV), totalizando el 46,2\% (Tabla 1). El gran número de disertaciones y tesis relacionadas al capítulo XXI de la CIE 10 está vinculado a 
Tabla 1. Distribución de disertaciones y tesis de los programas de postgrado de la EERP-USP del 2007 al 2009 según los capítulos de la CIE 10, Ribeirão Preto 2011.

\begin{tabular}{lrr}
\hline Grupo de Causas (capítulos de la CIE 10) & $\mathrm{n}$ & $\%$ \\
\hline Factores que afectan el estado sanitario y el contacto con los servicios de salud (capítulo XXI) & 42 & 17,6 \\
Trastornos mentales y del comportamiento (capítulo V) & 40 & 16,8 \\
Enfermedades endocrinas, nutricionales y metabólicas (capítulo IV) & 28 & 11,8 \\
Neoplasmas [tumores] (Capítulo II) & 21 & 8,8 \\
Gravidez, parto y puerperio (capítulo XV) & 18 & 7,6 \\
Algunas enfermedades infecciosas y parasitarias (capítulo I) & 18 & 7,6 \\
Enfermedades del sistema circulatorio (capítulo IX) & 15 & 6,3 \\
Enfermedades del sistema respiratorio (capítulo X) & 10 & 4,2 \\
Lesiones, intoxicaciones y algunas otras consecuencias de causas externas (capítulo XIX) & 7 & 2,9 \\
Otros & 39 & 16,4 \\
\hline Total & $238^{\star}$ & 100 \\
\hline
\end{tabular}

* tres disertaciones/tesis estaban relacionadas a dos capítulos de la CIE 10.

la categoría específica "personas con riesgos potenciales de salud relacionadas con circunstancias socioeconómicas y psicosociales”. Esa categoría incluye: exposición ocupacional a los factores de riesgo, problemas relacionados con el ambiente físico, problemas relacionados con la habitación y con las condiciones económicas y otros problemas relacionados con el grupo primario de apo- yo, incluso situación familiar (11).

En el 2007, los principales grupos de causas de mortalidad accedidos en el DATASUS fueron, respectivamente, enfermedades del sistema circulatorio (capítulo IX), neoplasmas [tumores] (capítulo II), causas externas de morbilidad y mortalidad (capítulo XX) y enfermedades del sistema respiratorio (capítulo X), totalizando el 67,3\% (Tabla 2).

Tabla 2. Distribución de la mortalidad general brasileña por grupos de causas en el 2007, Ribeirão Preto 2011.

\begin{tabular}{lcc}
\hline Grupo de Causas (capítulos de la CIE 10) & $\mathrm{n}$ & $\%$ \\
\hline Enfermedades del sistema circulatorio (capítulo IX) & 308.466 & 29,4 \\
Neoplasmas [tumores] (Capítulo II) & 161.491 & 15,4 \\
Causas externas de morbilidad y mortalidad (capítulo XX) & 131.032 & 12,5 \\
Enfermedades del sistema respiratorio (capítulo X) & 104.498 & 10 \\
Síntomas y observaciones clínicas o de laboratorio anormales no clasificados en otra parte & 80.244 & 7,7 \\
(capítulo XVIII) & 61.860 & 5,9 \\
Enfermedades endocrinas, nutricionales y metabólicas (capítulo IV) & 53.724 & 5,1 \\
Enfermedades del sistema digestivo (capítulo XI) & 45.945 & 4,4 \\
Algunas enfermedades infecciosas y parasitarias (capítulo I) & 26.898 & 2,6 \\
Enfermedades del feto y del recién-nacido (capítulo XVI) & 73.666 & 7 \\
Otras causas & 1.047 .824 & 100 \\
\hline Total & & \\
\hline
\end{tabular}

Fuente: DATASUS. 
La comparación de los temas de las disertaciones y tesis con los datos de mortalidad general muestran que la producción se relaciona con las causas de fallecimiento más frecuentes (Figura 1). De los nueve temas principales y causas de mortalidad general, cinco fueron similares en ambos grupos (capítulos IV, II, I, IX y X).
Los principales grupos de causas de morbilidad hospitalaria encontrados fueron embarazo, parto y puerperio (capítulo XV), enfermedades del sistema respiratorio (capítulo X) y enfermedades del sistema circulatorio (capítulo IX), representando casi la mitad $(45,8 \%)$ de todas las causas de morbilidad hospitalaria en el 2007 en Brasil (Tabla 3).

Figura 1. Comparación del porcentaje de disertaciones y tesis con el porcentaje de mortalidad general de Brasil según capítulos de la CIE 10*, Ribeirão Preto 2011.
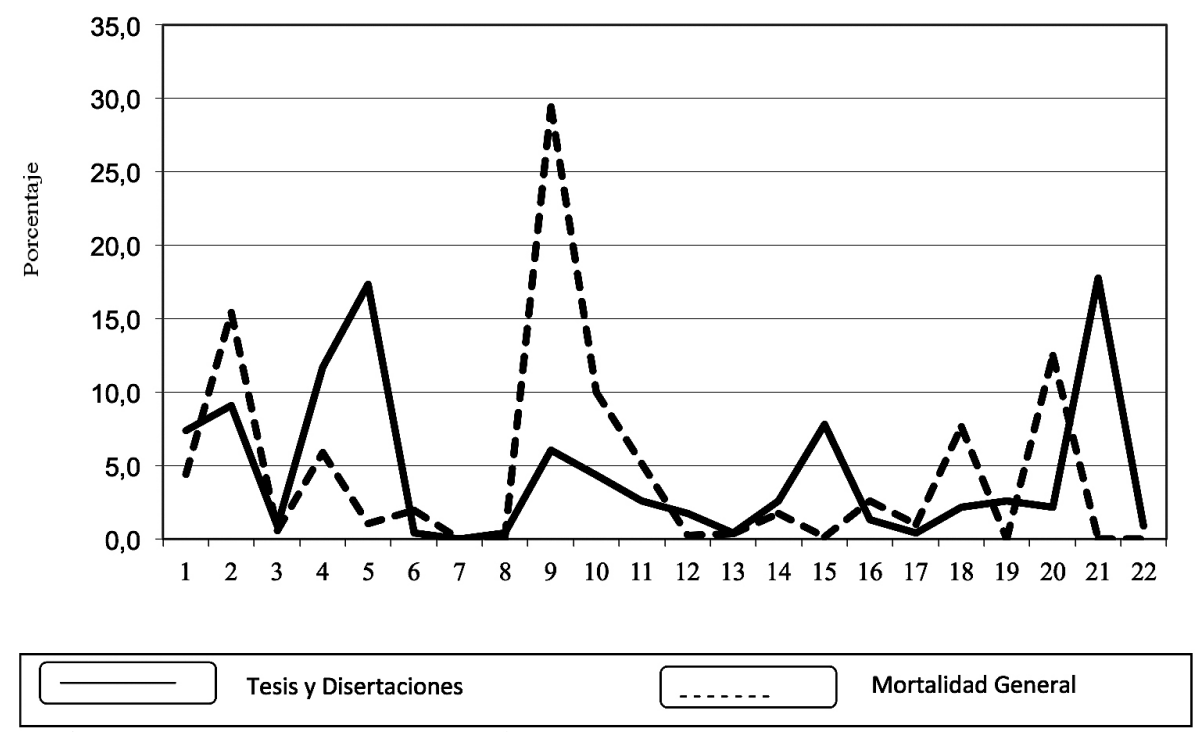

* Los capítulos de la CIE 10 fueron mostrados en números arábigos para facilitar la visualización.

Tabla 3. Distribución de la morbilidad hospitalaria brasileña por grupos de causas en el 2007, Ribeirão Preto 2011.

\begin{tabular}{lrr}
\hline Grupo de Causas (capítulos de la CIE 10) & $\mathrm{n}$ & $\%$ \\
\hline Embarazo, parto y puerperio (capítulo XV) & 2.486 .305 & 21,9 \\
Enfermedades del sistema respiratorio (capítulo X) & 1.550 .295 & 13,7 \\
Enfermedades del sistema circulatorio (capítulo IX) & 1.157 .509 & 10,2 \\
Enfermedades del sistema digestivo (capítulo XI) & 996.335 & 8,8 \\
Algunas enfermedades infecciosas y parasitarias (capítulo I) & 915.763 & 8,1 \\
Lesiones, intoxicaciones y algunas otras consecuencias de causas externas (capítulo XIX) & 831.051 & 7,3 \\
Neoplasmas [tumores] (capítulo II) & 640.325 & 5,7 \\
Trastornos mentales y del comportamiento (capítulo V) & 290.079 & 2,6 \\
Enfermedades endocrinas, nutricionales y metabólicas (capítulo IV) & 281.575 & 2,5 \\
Otras causas & 2.180 .859 & 19,2 \\
\hline TOTAL & 11.330 .096 & 100 \\
\hline
\end{tabular}

Fuente: DATASUS. 
Figura 2. Comparación del porcentaje de disertaciones y tesis con el porcentaje de morbilidad hospitalaria según capítulos de la CIE 10*, Ribeirão Preto 2010.

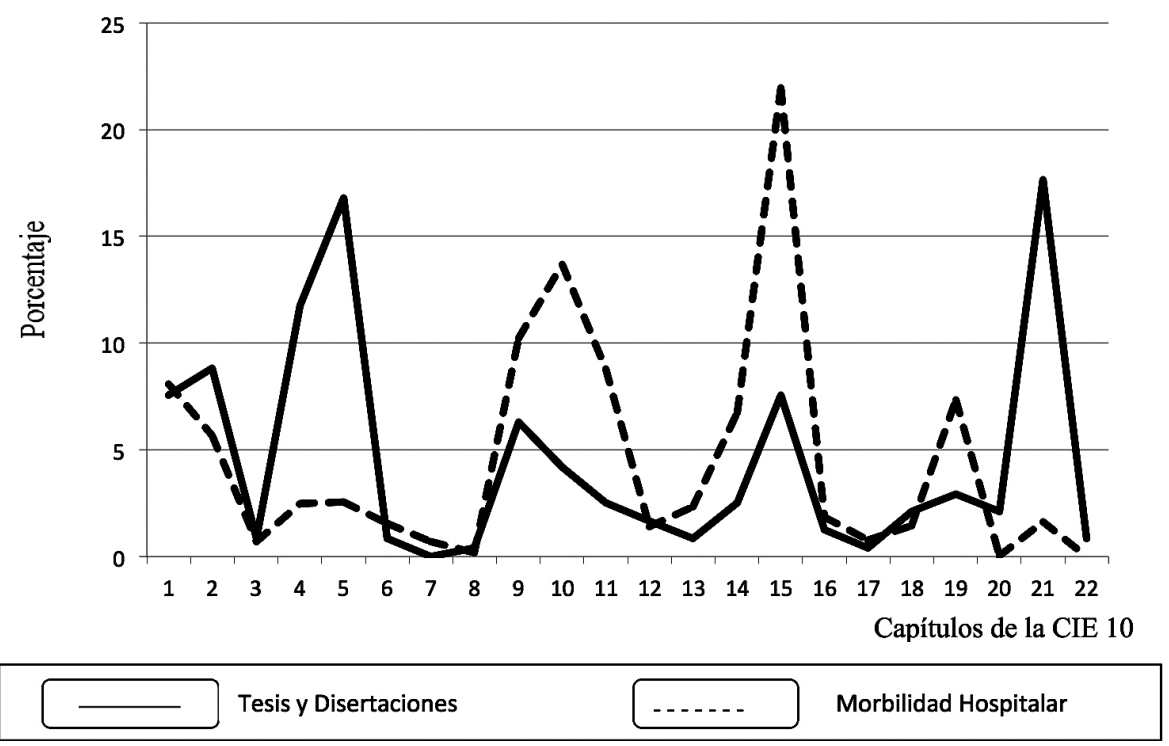

* Los capítulos de la CIE 10 fueron mostrados en números arábigos para facilitar la visualización.

La comparación de los temas de las disertaciones y tesis con los datos de morbilidad hospitalaria mostraron una fuerte relación de la producción con las mayores causas de morbilidad (Figura 2). De los nueve principales temas y causas de morbilidad en Brasil, ocho coincidieron (capítulos V, IV, II, XV, I, IX, X y XIX).

\section{DISCUSIÓN Y CONCLUSIÓN}

La producción científica de los cursos de maestría y doctorado en enfermería en Brasil es uno de los principales parámetros para evaluar los programas de postgrado. Esa producción debe priorizar las necesidades de la población y de los servicios de salud (15).

Para orientar la producción de conocimientos, bienes materiales y procesuales en las áreas prioritarias para el desarrollo de las políticas sociales fue creada la Agenda $\mathrm{Na}$ cional de Prioridades de Investigación en
Salud (ANPPS), que tiene como presupuesto respectar las necesidades nacionales y regionales de salud, de acuerdo con los principios del SUS (16).

El área de enfermería de la CAPES propone 11 líneas de investigación: fundamentos teórico-filosóficos del cuidar en salud y enfermería; tecnología en salud y enfermería, ética en salud y enfermería, historia de la enfermería, proceso de cuidar en salud y enfermería, salud y calidad de vida, políticas y práctica en salud y enfermería, políticas y práctica de educación y enfermería, producción social y trabajo en salud y enfermería, gestión de los servicios de salud y de enfermería e información/comunicación en salud y enfermería. Esas son categorizadas en tres grandes áreas: profesional, asistencial y organizacional (15).

El principal tema por grupo de causas encontrado en este estudio (17,6\%) fue relacionado al capítulo XXI de la CIE 10, que abarca una de las áreas tradicionales de conocimiento producido por la enfermería: salud 
del trabajador. Este tema está relacionado a la línea de investigación propuesta por el área de enfermería de la CAPES "producción social y trabajo en salud y enfermería" y es una de las prioridades de investigación de la ANPPS (salud, ambiente, trabajo y bioseguridad) (16).

El gran número de disertaciones y tesis relacionadas al capítulo V de la CIE 10 se justifica por la presencia del Programa Enfermería Psiquiátrica, específico y único entre los programas de postgrado de enfermería brasileña para esta temática. La distribución de los resúmenes publicados en los catálogos del Centro de Estudios e Investigación en Enfermería (CEPEn) en el área de salud mental de 1979 al 2007 mostró predominancia de estudios producidos por la USP. De los 280 resúmenes estudiados, 180 eran de esa institución (17). Se destaca que la salud mental es una importante prioridad de investigación según la ANPPS (16).

El capítulo IV se destacó entre los temas de las tesis y disertaciones producidas en la EERP-USP, principalmente debido a la categoría específica "diabetes mellitus", incluida entre las prioridades de la ANPPS (16).

Algunos temas encontrados en las disertaciones y tesis no están relacionados con cualquier capítulo de la CIE 10, pero representan prioridades de investigación en la ANPPS, tales como gestión, enseñanza, ancianos, urgencia y emergencia, políticas de salud, ética y bioética, medio ambiente, promoción de la salud y desarrollo de software (16). La investigación en el área de enfermería abarca diversos aspectos y muchas veces no está relacionada a una enfermedad o un problema de salud específico. En un estudio referente a las producciones sobre el cuidado de enfermería, predominaron los temas de gestión de enfermería, humanización del cuidado y cuidado al niño hospitalizado (18).

En la comparación entre los datos de mortalidad general, morbilidad hospitalaria y los temas de las tesis y disertaciones producidas, se destacaron los capítulos II, I, IX y
$\mathrm{X}$, con enfoque no sólo en la patología, sino también en los aspectos psicosociales. Eso es compatible con el saber holístico de la enfermería, que está en consonancia con las prioridades de la ANPPS (16).

Otro capítulo importante para morbilidad hospitalaria y producción de disertaciones y tesis fue el de "embarazo, parto y puerperio", lo que demuestra la importancia de este tema, constituyendo un reto para la sociedad y políticas públicas en Brasil, resultado de las consecuencias del parto tanto para la madre como para los recién nacidos.

Un estudio, cuyo objetivo fue caracterizar los egresos y tesis defendidas del Programa Interunidades de Doctorado en Enfermería de la EE-USP y EERP-USP entre 1998 y 2008, investigó las tesis producidas respecto a las áreas temáticas. Las áreas con mayor número de tesis vinculadas fueron salud de la mujer $(20,5 \%)$ y salud del adulto y anciano $(13,2 \%)$. Esos resultados mostraron que, en este programa, la producción científica está relacionada con los indicadores de mortalidad general y morbilidad hospitalaria nacional. Se destaca que la temática "salud del adulto y anciano" es muy amplia, lo que dificulta la evaluación específica conforme a los capítulos de la CIE 10(2).

El desarrollo del conocimiento en la educación de postgrado es influenciado por indicadores sociales, económicos, políticos, demográficos y por el contexto del sistema de salud. Así, el impacto de las producciones debe favorecer a la población, la práctica profesional y las políticas de salud del país (5).

La comparación de los temas de las disertaciones y tesis producidas por los Programas de postgrado en enfermería de la EERP-USP con los datos de mortalidad general y morbilidad hospitalaria mostraron que existe relación de la producción con esos indicadores. Se destacaron las enfermedades endocrinas, nutricionales y metabólicas, neoplasmas (tumores), algunas enfermedades infecciosas y parasitarias, enfermedades del sistema circu- 
latorio y enfermedades del sistema respiratorio.

\section{Limitaciones del estudio}

Se encontró dificultad en la clasificación de las disertaciones y tesis según los capítulos de la CIE 10, principalmente cuando no existía relación con una enfermedad específica. Una gran cantidad de disertaciones y tesis estuvo relacionada al capítulo XXI de la CIE 10, el que no posee relación directa con los datos de mortalidad general y morbilidad hospitalaria.

Los datos de mortalidad demandan corrección de la subnumeración de fallecimientos captados por el SIM, especialmente en las regiones Norte y Nordeste. Los datos presentan restricción de uso siempre que ocurre una alta proporción de fallecimientos sin atención médica o por causas mal definidas (7).

Para los datos de morbilidad hospitalaria no se consideraron las unidades que no tienen vínculo con el SUS. Este indicador puede ser influenciado por el recuento acumulativo de internaciones de un mismo paciente, debido a la misma causa, durante el período analizado (7).

\section{REFERENCIAS}

1. Erdmann AL. Editorial. Texto contexto enferm. 2006; 15(4): 547-548.

2. Garbin LM, Azevedo ALCS, Silva LTR, Laus AM, Chaves LDP, Gualda DMR, et al. Programa Interunidades de Doutoramento em Enfermagem da Universidade de São Paulo: caracterização dos egressos e teses defendidas em uma década. Rev Lat Am Enfermagem. 2010; 18(5): 841-8.

3. Almeida MCP, Silva EM, Villa TCS, Assis MMA, Kemura MLR. O conhecimento produzido no programa de mestrado de enfermagem em saúde pública da Escola de Enfermagem de Ribeirão Preto-USP e suas relações com as práticas de saúde. Rev Lat Am Enfermagem. 2000; 8(5): 916.

4. Ministério da Educação Brasil, Coordenação de Aperfeiçoamento de Pessoal de Nível Superior (CAPES). Relação de cursos recomendados e reconhecidos. [Internet]. Brasilia: CAPES; 2010 [citado 1 nov 2010]. Disponível em: http://conteudoweb.capes.gov.br

5. Ministério da Educação Brasil, Coordenação de Aperfeiçoamento de Pessoal de Nível Superior (CAPES). Resultados Finais - Avaliação trienal 2010 [Internet]. Brasilia: CAPES; 2010 [citado 1 nov 2010]. Disponível em: http://trienal.capes.gov.br

6. Souza MFM. Dos dados a política: a importância da informação em saúde. Epidemiol. Serv. Saúde. 2008; 17(1): 5-6.

7. Rede Interagencial de Informação para a Saúde - RIPSA. Indicadores básicos para a saúde no Brasil: conceitos e aplicações [Internet]. 2a ed. Brasília: Organização Pan-Americana da Saúde; 2008 [citado 1 nov 2010]. 349 p. Disponível em: http://tabnet.datasus.gov.br/tabdata/ livroidb/2ed/indicadores.pdf

8. Prado ML do, Kuerten RP, Schubert BVM, Reibnitz KS, Waterkemper R, Coelho GD. Produção de conhecimento em um curso de mestrado em enfermagem no Brasil. Cienc. enferm. 2011; XVII(3): 43-50.

9. Polit DF, Beck CT, Hungler BP. Fundamentos de Pesquisa em Enfermagem. Métodos, avaliação e utilização. 5a ed. Porto Alegre: Artmed; 2004. 487 p.

10. Ministério da Educação Brasil. Coordenação de Aperfeiçoamento de Pessoal de Nível Superior (CAPES) Caderno Avaliação. [Internet]. Brasilia: CAPES; 2010 [citado 1 nov 2010]. Disponível em: http://conteudoweb.capes.gov.br/conteudoweb/CadernoAvaliacaoServlet

11. Organização Mundial da Saúde. Classificação Estatística Internacional de 
Doenças e Problemas Relacionados à Saúde (CID 10) [Internet]. 2008. Décima revisão. São Paulo (SP): Organização Mundial da Saúde; 2008 [citado 1 nov 2010]. Disponível em: http://www.datasus.gov.br/cid10/v2008/cid10.htm

12. Ministério da Saúde Brasil. Departamento de Informática do Sistema Único de Saúde. Informações de Saúde [Internet]. Brasília: Ministério da Saúde; 2007 [citado 1 nov 2010]. Disponível em: http:// tabnet.datasus.gov.br.

13. Motta DN, Lopes LAB, Pereira MG. Estatísticas vitais do distrito federal: o perfil da mortalidade em 2008. Brasília Med. 2010; 48(2): 161-6.

14. Conselho Nacional de Saúde, Comissão Nacional de Ética em Pesquisa. FAQ - Perguntas e respostas: Questões Gerais [Internet] Brasil: CONEP; 2012 [citado 10 mar 2012]. Disponível em: http://conselho. saude.gov.br

15. Rodrigues RAP, Erdmann AL, Silva
IA, Fernandes JD, Araújo TL, Vianna LAC, et al. Educação do doutorado em enfermagem no Brasil. Rev Lat Am Enfermagem. 2008; 16(4): 665-71.

16. Ministério da Saúde Brasil, Secretaria de Ciência, Tecnologia e Insumos Estratégicos, Departamento de Ciência e Tecnologia. Agenda Nacional de Prioridades de Pesquisa em Saúde (ANPPS) [Internet]. 2a ed. Brasília: Ministério da Saúde; 2008 [citado 1 nov 2010]. 68 p. Disponível em: bvsms.saude. gov.br/bvs/publicacoes/AGENDA_ PORTUGUES_MONTADO.pdf

17. Silva KVLG, Almeida ANS, Monteiro ARM, Silveira LC, Fialho AVM, Moreira TMM. Análise das dissertações e teses de enfermagem sobre saúde mental, Brasil, 1979-2007. Rev Lat Am Enfermagem. 2010; 18(5): 1031-1038.

18. Cogo ALP, Crossetti MGO. Tendências da pesquisa sobre o cuidado de enfermagem. Rev Gaucha Enferm. 2010; 31(1): 8 . 